\title{
Adipocytokine, leptin and resistin in combination with quantitative visceral adiposity as predictors of colorectal neoplasm: A Case- Control Study
}

\author{
Engy Shafik ${ }^{1}$, Dina Mohareb ${ }^{2}$, Helal Hetta ${ }^{3,4}$, Salah Khallaf ${ }^{5}$, Nessren Abdel-Rady ${ }^{6}$, Zainab Gaber ${ }^{7}$, Mohamed \\ Abu Rahma7, Wael Abbas ${ }^{8}$, Haisam Atta ${ }^{9}$ \\ ${ }^{1}$ Department of Clinical Pathology, South Egypt Cancer Institute, Assiut University, Egypt \\ 2Department of Clinical Pathology, Faculty of Medicine, Assiut University, Egypt \\ ${ }^{3}$ Department of Medical Microbiology and Immunology, Faculty of Medicine, Assiut University, Egypt \\ ${ }^{4}$ Department of Internal Medicine, University of Cincinnati College of Medicine, Cincinnati, OH, USA \\ ${ }^{5}$ Department of Medical Oncology, South Egypt Cancer Institute, Assiut University, Egypt \\ ${ }^{6}$ Department of Physiology, Faculty of Medicine, Assiut University, Egypt \\ ${ }^{7}$ Department of Tropical Medicine, Faculty of Medicine, Assiut University, Egypt \\ ${ }^{8}$ Department of Internal Medicine, Faculty of Medicine, Assiut University, Egypt \\ ${ }^{9}$ Department of Radiology, South Egypt Cancer Institute, Assiut University, Egypt
}

\section{III}

\section{ABSTRACT}

Background: Substantial evidence has supported the association between adipocytokines and many types of cancers. However, the results in the setting of colorectal cancer (CRC) remain conflicting. Aim: This study aims to evaluate the predictive power of adipocytokines (Leptin and Resistin) levels and abdominal fat distribution in the diagnosis of colorectal cancer. Methods: Eighty participants were recruited then divided into three groups based on endoscopic and histopathological examination; 30 patients with colon cancer, 25 with colonic adenoma, and 25 controls. Serum adipocytokines (Leptin and Resistin) levels were measured, and the quantitative fat analysis was calculated based on an abdominal CT scan. Results: Multivariable logistic regression revealed that two predictors (leptin and Resistin) of CRC development could be used. Visceral/superficial fat rate was the most significant predictor among the parameters of quantitative fat analysis. Also, psoas muscle and visceral fat volume showed non-significant differences among the study groups. Conclusion: For the diagnosis of colorectal cancer, Leptin and Resistin were valuable independent predictors, while quantitative fat analysis parameters failed to be; the most important of these parameters in prediction was Visceral/superficial fat rate.

Keywords: colorectal cancer; Leptin; Resistin; fat analysis

Editor-in-Chief: Prof. M.L. Salem, PhD - Article DOI: 10.21608/JCBR.2020.28094.1029

\section{INTRODUCTION}

It is believed that oncologists need validated predictive factors for risk stratification of colorectal neoplasm patients (Hopkins et al., 2018). Many theories were postulated to understand the contributing factors in the development of colorectal cancer (CRC), among them; the role of feeding habits, lifestyle, obesity, as well as genetic and environmental factors interactions (Erarslan et al., 2009; Hopkins et al., 2018).
Patients' body composition and obesity have significant contribution with documented association between cancer incidence and obesity (Erarslan et al., 2009; Paik et al., 2009; Renehan et al., 2010; Khandekar et al., 2011), and previously increased cancer-associated mortality (Calle et al., 2003). Body composition could be evaluated by imaging using computed tomography (CT), allowing quantitative assessment of visceral obesity and alteration in muscle volume (Hopkins et al., 2018). 
The increase in abdominal fat contributed in molecular mechanism of CRC development via the production of several factors (adipocytesecreted hormones and pro-inflammatory cytokines) with a role in chronic inflammatory state related to obesity (Tulubas et al., 2013). Moreover, among most commonly accused adipocyte-secreted hormones are Leptin and Resistin in addition to adiponectin and ghrelin (Tulubas et al., 2013).

Leptin was considered as a product of human obese gene, and its implication in pathogenesis of several types of obesity-related cancers (PazFilho et al., 2011; Tudek and Speina, 2012). Furthermore, the effect of Leptin depends on its role in regulation of intracellular pathways and neoangiogenesis (Drew, 2012), as inflammatory mediator that stimulates secretion of other inflammatory factors (Paz-Filho et al., 2011). Resistin plays a similar role in oncogenesis and chronic inflammation, in addition to its role in cancer development, where it is produced by adipose tissue and share in activation of monocytes as part of generalized inflammatory process (Gonullu et al. 2010). The current study aims to evaluate association between adipocytokines (Leptin and Resistin) levels and abdominal fat distribution, with the diagnosis of colorectal cancer.

\section{MATERIAL AND METHODS}

This is a prospective case-control study. Ethical approval was obtained from local Institutional review board (IRB) [SECI-IBR IORG0006563] before execution. All participants received a written consent form explicit and indicative of the purpose of study, allowing participants to approve or decline participation without obligation. Furthermore, assurance of the participants' confidentiality and anonymity was guaranteed by assigning each participant a code number for analysis only. The study was not based on incentives or rewards for participants. Sample size calculation was conducted using G*Power-3 software (Faul et al., 2007). Calculated minimum sample of 75 participants ( 25 CRC, 25 adenoma, 25 control) is required to establish effect size of 0.2 in mean serum adipocytokines levels, with an error probability of 0.05 and $80 \%$ power on a two-tailed test.

\section{Study Procedure}

From June 2018 to June 2019, all patients referred to the endoscopy units, Assiut University Hospitals and South Egypt Cancer Institute, Assiut, Egypt, and were eligible for the study, were recruited to fulfill the required sample size. Inclusion criteria were; patients diagnosed as CRC and colonic adenoma by endoscopy and confirmed by histopathology, and control group referred to colonoscopy for lower GIT symptoms, and their colonoscopy results revealed normal endoscopic findings and normal biopsy. Exclusion criteria included: Synchronous malignancies, prior treatment by chemotherapy or radiotherapy, major surgeries during the six months before recruitment, inflammatory bowel disease, previous gastrointestinal surgery or even patient refusal to participate in study. A detailed history and clinical examination of all participants were done. This included symptoms and signs suggestive of colorectal neoplasm, Family history of the CRC was obtained.

Out of 100 eligible participants, 16 patients refused to be involved in the study after the explanation of the aim and steps of research. Another four patients did not perform the abdominal CT imaging due to suspicions of early pregnancy. Eighty participants were included, then divided into three groups according to the endoscopic findings and histopathological analysis of endoscopic biopsy; the $1^{\text {st }}$ group included 30 patients with pathologically-proven CRC. The $2^{\text {nd }}$ group included 25 patients with colonic adenomas. The $3^{\text {rd }}$ group (control group) included 25 patients confirmed to be free from any colonic neoplasm, as proved by endoscopic and histopathological assessment.

Also, a biopsy was performed for the control group to exclude microscopic neoplastic lesions or chronic inflammatory bowel diseases (Figure 1). All participants were subject to laboratory tests and imaging studies with a mean of 3-5 days between the two investigations after their endoscopic evaluation. Routine laboratory tests performed included blood samples, tumor markers (CA-19-9 and CEA) levels, in addition to serum adipocytokines (Leptin and Resistin) levels. 


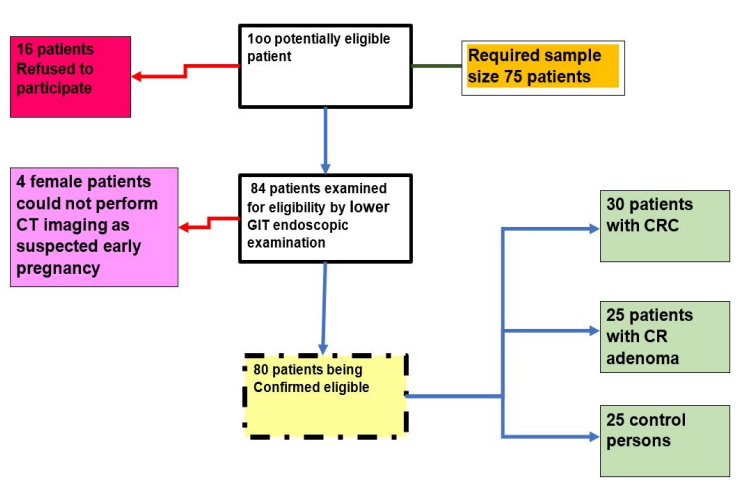

Figure 1. Consort diagram of the participants.

Adipocytokines measurements Three milliliters of blood were acquired via a median cubital vein puncture from each participant into vacutainer tube (BD, Franklin Lakes, NJ, USA). Whole blood was allowed to clot for 20 minutes at $37^{\circ} \mathrm{C}$ and centrifuged at $2000 \mathrm{rpm}$ for 10 minutes. Serum was aliquoted and stored at $-20^{\circ} \mathrm{C}$ until analyzed. The routine tumor markers (CEA and CA 19.9) were conducted by immunoenzymatic (sandwich) assay using Beckman Coulter-Access 2 Analyzer SN: 510552. Leptin and Resistin levels were measured by ELISA using Human Leptin ELISA kit; CELL BIOLABS, INC, cat no. MET5057. Human Resistin ELISA kit; AssayMaxTM cat no.ER1001-1. Plate reading was carried out using Humareader HS, SN.: 501018015 HBSE. Calculation of Leptin results was done by preparing new standards by diluting Leptin standards stock tubes and preparation of Leptin standard curve. Calculation of Resistin results was done by making triplicate standard points by serially diluting from the standard stock solutions. A freshly prepared standard curve was used at each time the assay was performed.

Imaging acquisition All CT scans were obtained on a 16-multidetector scanner (Bright speed CT, GE Medical Systems). Patients' position within the machine was supine with head first. Scans were obtained from supra-diaphragmatic level to below symphysis pubis with automated tube current modulation (mA); $120 \mathrm{kVp}$; pitch 1.3 (Atta et al., 2016)

Quantitative fat analysis Based on non-contrast MSCT of abdomen, studies were acquired from the local PACS system and 3D synapse workstation employing 3D fat analysis software (synapse, Fujifilm) (Figure 2). Starting analysis just above diaphragm and reaching down to level of symphysis pubis (Ohshima et al., 2008).

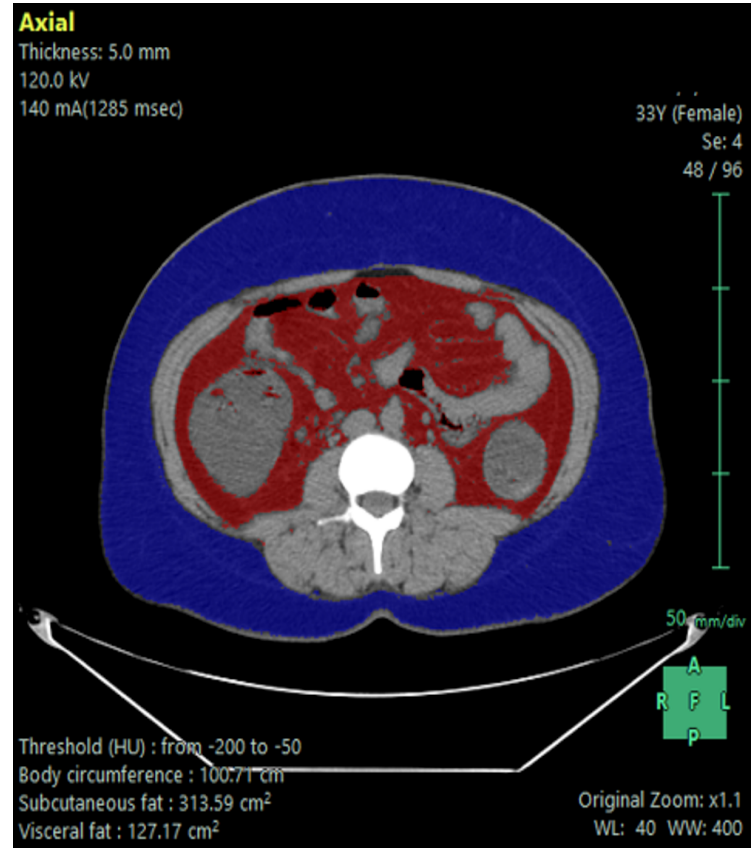

Figure 2. Semi-automatic abdominal fat analysis (subcutaneous fat is coded in blue color while visceral fat is coded in red).

Software creates an analysis range, preparation of this range for analysis was done through adjustment of the constructed contour lines of abdominal walls and psoas muscle if necessary. Mask borders were re-drawn to exclude lung bases from the analysis range.

Quantitative analysis includes; an automated calculation of the visceral and subcutaneous fat volumes via 3D analysis of each entity. Moreover, the software provides the fat rate within the analysis range according to the equation of dividing the sum of visceral and subcutaneous fat by the volume of abdominal field.

Graphical display of the analysis results, in addition to the generation of an automatic report after manual insertion of the patients' weight and height, was provided. The report includes; the fore-mentioned calculated data, visceral/subcutaneous $(\mathrm{V} / \mathrm{S})$ fat rate, psoas muscle volume, and body mass index (BMI) (Figure 3 ). Also, the software creates an obesity index (Figure 4) of three points score ranging within too thin, normal, and too fat (Summers et al., 2012).

Statistical analysis: The collected data were verified, coded, and analyzed using IBMSPSS/PC/VER-21). Descriptive statistics: mean, 
standard deviation, median, range, frequencies, percentage calculated. Test of significance: Chisquare test was calculated to compare the difference in distribution of frequencies among different groups. For continuous variables with more than two categories, ANOVA test was calculated to test the mean differences of the data that follow a normal distribution. The posthoc test was done using Bonferroni corrections for pairwise comparisons. Multivariable logistic regression analysis is calculated to investigate predictive power of body fat composition determinants of the studied Cohort on the CRC and Adenoma (Odds Ratio -OR-, 95\% confidence interval $-95 \% \mathrm{Cl}$ - and Likelihood Ratio Test p-value -LRT-). ROC analysis was calculated to test the validity of the different predictors against biopsy results. Validity statistics (sensitivity, specificity, positive and negative predictive value -PPV \& NPV- and accuracy) calculated. A significant $p$ value was considered when it is $<0.05$.

\section{RESULTS}

As regards of demographic data, the mean ages \pm standard deviation were $45.23 \pm 19.6$ for CRC patients, $52.40 \pm 17.8$ for patients with adenoma, and $55.12 \pm 15.8$ for the control group. Males were slightly more common than females in CRC arm (16/14) and in adenoma arm $(15 / 10)$, while the reverse occurred in control participants (12/13). Only 2 cases of CRC had a positive family history of CRC, while only one case with adenoma had a first close-relative having cancer colon. In patients with CRC, the most common symptom was abdominal pain (21\%) followed by chronic constipation (15\%), then bloody stool (8\%). These symptoms were relatively rare either in patients with adenoma or in the control group. No significant differences were encountered within the three groups regarding age or sex. On the other hand, $B M I$ shows no significant difference between $C R C$ and control groups $(P=0.488)$, although there were differences among the three groups, i.e., adenoma group had lower BMI compared with the other two groups (Tables 1 and 2).

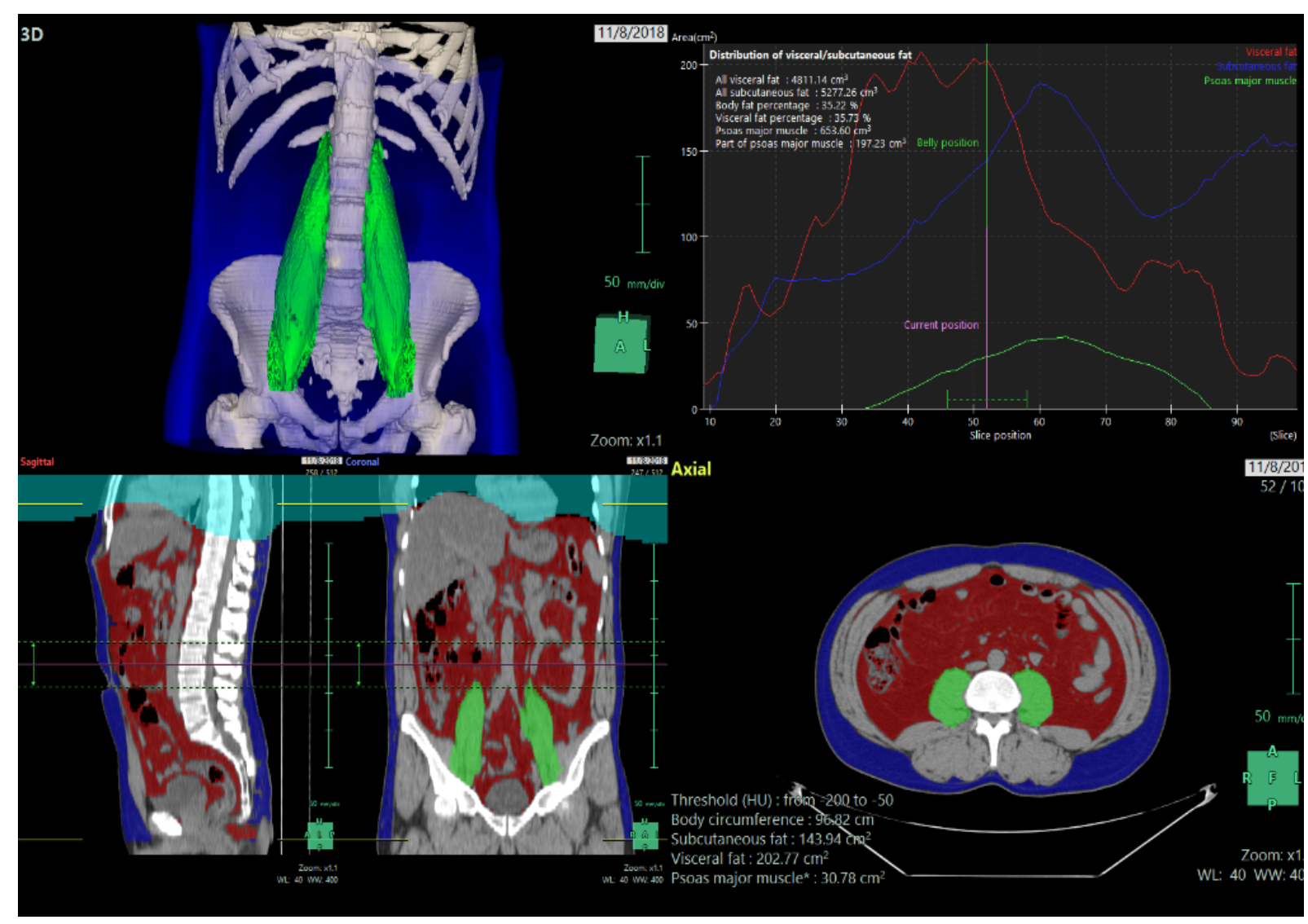

Figure 3. Quantitative fat analysis of the volume of psoas muscle coded in green color and standard report that is autocreated, demonstrating quantitative data on different parameters and obesity index in automated report data. 


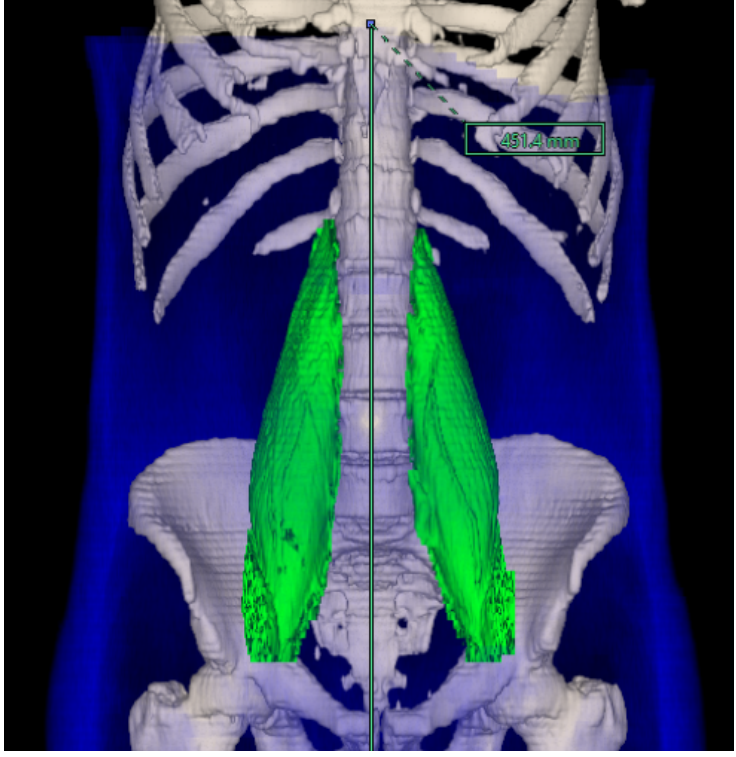

Measurments:

- Visceral fat volume: $4820.16 \mathrm{~cm}^{3}$

- Subcutaneous fat volume: $5197.86 \mathrm{~cm}^{3}$

- Fat rate in body: $35.26 \%$

- Visceral fat volume rate: $35.66 \%$

- BMI: 24.38

- 3D V/S rate: 0.93

- Psoas major muscle volume: $653.60 \mathrm{~cm}^{3}$ Total Result:

The obesity index of BMI: Normal

Your ideal weight: $79.42 \mathrm{~kg}$

(Between 66.78 and $90.25 \mathrm{~kg}$ is normal weight

\section{Too Thin Normal Too Fat}

Figure 4. Standard report that is auto-created, demonstrating quantitative data on different parameters and obesity index in automated report data.

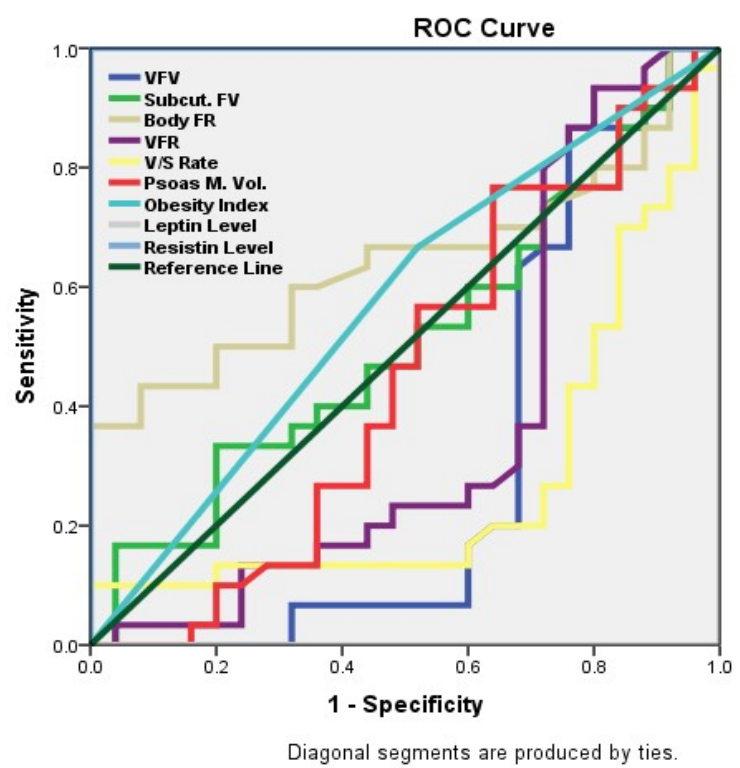

Figure 5. ROC curve for colorectal cancer prediction by body fat composition determinants.
The most common site of malignant lesions was the sigmoid colon in $26.6 \%$ of malignant cases, while the histopathological types vary, with the most encountered variant was moderately differentiated adenocarcinoma in 17 patients (57\% of malignant cases) (Table 3). The tumor markers had higher values as CA19-9 (24.6 \pm 10.9 $\mathrm{U} / \mathrm{ml}$ ) in CRC patients with significant difference among the different study groups $(P<0.001)$. Also, CEA showed quite higher values in the CRC group of patients in this study $(6.8 \pm 1.7 \mathrm{ng} / \mathrm{ml})$, with a significant difference $(P<0.001)$ among the study groups, and in between CRC and control group.

The diagnostic performance of the different CT body fat parameters revealed a significant difference among three groups when handling visceral fat volume, $\mathrm{V} / \mathrm{S}$ fat rate, and psoas muscle volume. Whereas, visceral fat volume shows significant value in differentiating between groups, although this parameter was not considered in differentiating CRC from adenoma groups. Furthermore, a statistically significant difference was encountered in $\mathrm{V} / \mathrm{S}$ fat rate and psoas muscle volume parameters between CRC and adenoma groups, as well as between adenoma and control groups.

Regarding the validity analysis of different parameters, $\mathrm{V} / \mathrm{S}$ fat rate shows $73 \%$ accurate differentiation between CRC from the other groups. Leptin and Resistin shows significant distinction between the three groups $(P<0.001)$, while Resistin did not have statistically differentiating power between adenoma and control groups. Leptin had a perfect accuracy $(100 \%)$ in differentiating CRC from the control group. Unlikely, the psoas muscle volume showed significant difference regarding differentiating CRC and adenoma in different adenoma from control. However, this parameter did not show proper power in the characterization of CRC from control, with an accuracy of about 55\% (Table 4 and Figure 5).

Among the different study parameters, two significant independent predictors of CRC were detected in the multivariable logistic regression model (Table 5); Leptin and Resistin. As with one $\mathrm{ng} / \mathrm{ml}$ increase in the level of Leptin, there was a $44 \%$ reduction in the risk of developing CRC ( $O R=0.556,95 \% \mathrm{Cl} 0.41-0.76, \mathrm{P}<0.001)$. On the 
other hand, with one $\mathrm{ng} / \mathrm{ml}$ increase in Resistin, there was a three-fold increase in probability of developing CRC among the study population. (OR $=3.13,95 \% \mathrm{Cl} 1.070-7.824, p=0.009)$. The other measures did not possess any significance regarding the prediction power.

\section{DISCUSSION}

Obesity is considered among the potential risk factors for oncogenesis(Nishii et al., 2001; Petridou et al., 2003; Calle and Thun, 2004) Many studies were directed towards understanding the physiology of obesity. As BMI fails to act as the best reflector of obesity, adipocytokines emerged as a biomarker for the neoplastic process and other metabolic syndromes to clarify the role of obesity involvement in oncogenesis (Petridou et al., 2000, 2003; Hsing et al., 2001; Miyoshi et al., 2003; Stattin et al., 2004; Goktas et al., 2005; Wei et al., 2005).

Although Leptin levels still have a debatable role in different studies. The current results showed a highly significant difference in serum Leptin concentration between different groups, and it was one of the most significant predictors, with perfect accuracy, even that Leptin levels were significantly lower in patients with CRC compared with the control subjects or when compared to the adenoma group.

Table 1. Baseline characteristics of the studied groups

\begin{tabular}{lcccc}
\hline & $\begin{array}{c}\text { CRC } \\
(\mathrm{n}=30)\end{array}$ & $\begin{array}{c}\text { Adenoma } \\
(\mathrm{n}=25)\end{array}$ & $\begin{array}{c}\text { Control } \\
(\mathrm{n}=25)\end{array}$ & P-value \\
\hline Age/years & $45.23 \pm 19.6$ & $52.40 \pm 17.8$ & $55.12 \pm 15.8$ & 0.861 \\
Sex (Male/Female) & $16 / 14$ & $15 / 10$ & $12 / 13$ & 0.695 \\
Weight/Kg & $63.13 \pm 18.6$ & $65.24 \pm 16.1$ & $75.80 \pm 13.2$ & 0.013 \\
BMI & $29.61 \pm 10.5$ & $23.83 \pm 7.1$ & $31.34 \pm 8.6$ & 0.009 \\
\hline
\end{tabular}

Table 2. Imaging and laboratory characteristics of the studied groups

\begin{tabular}{lcccc}
\hline & $\begin{array}{c}\text { CRC } \\
(\mathrm{n}=30)\end{array}$ & $\begin{array}{c}\text { Adenoma } \\
(\mathrm{n}=25)\end{array}$ & $\begin{array}{c}\text { Control } \\
(\mathrm{n}=25)\end{array}$ & P-value \\
\hline V-S Fat Rate & $0.90 \pm 0.3$ & $6.13 \pm 3.0$ & $0.83 \pm 0.4$ & 0.042 \\
Visceral Fat Vol. $\left(\mathrm{cm}^{3}\right)$ & $1.65 \pm 0.2$ & $2.46 \pm 0.5$ & $3.76 \pm 0.6$ & 0.005 \\
Superficial Fat Vol. $\left(\mathrm{cm}^{3}\right)$ & $6.02 \pm 1.1$ & $18.77 \pm 1.2$ & $12.04 \pm 7.3$ & 0.089 \\
Body Fat Rate & $44.65 \pm 20.1$ & $34.18 \pm 18.2$ & $35.93 \pm 13.4$ & 0.090 \\
Visceral Fat Rate & $26.33 \pm 10.3$ & $26.24 \pm 11.7$ & $32.49 \pm 13.8$ & 0.182 \\
Psoas Muscle Vol./cm ${ }^{3}$ & $338.21 \pm 23.1$ & $247.84 \pm 37.8$ & $380.14 \pm 35.4$ & 0.017 \\
Obesity Index & & & & \\
$\bullet \quad$ Too Thin & $0(0 \%)$ & $0(0 \%)$ & $0(0 \%)$ & 0.516 \\
$\bullet \quad$ Normal & $10(33.3 \%)$ & $11(44 \%)$ & $12(48 \%)$ & \\
$\bullet \quad$ Too Fat & $20(66.7 \%)$ & $14(56 \%)$ & $13(52 \%)$ & \\
Leptin (ng/ml) & $53.18 \pm 9.6$ & $63.03 \pm 4.4$ & $73.89 \pm 3.2$ & $<0.001$ \\
Resistin (ng/ml) & $18.86 \pm 2.6$ & $10.30 \pm 1.5$ & $9.55 \pm 1.4$ & $<0.001$ \\
CA 19-9 (U/ml) & $24.63 \pm 10.9$ & $5.04 \pm 2.0$ & $3.85 \pm 1.8$ & $<0.001$ \\
CEA ng/ml & $6.81 \pm 1.7$ & $1.47 \pm 0.7$ & $1.36 \pm 0.9$ & $<0.001$ \\
\hline
\end{tabular}

Table 3. Endoscopic site of the malignant lesion and the histopathological types

\begin{tabular}{lll}
\hline & & $\begin{array}{l}\text { Number of cases } \\
\text { (\% of malignant cases) }\end{array}$ \\
\hline Site & Caecum & $7(23 \%)$ \\
& Descending colon & $5(16.6 \%)$ \\
& Sigmoid & $8(26.6 \%)$ \\
& Upper rectum & $4(13.3 \%)$ \\
& Mid-Low rectum & $6(20 \%)$ \\
\hline Pathological type & Mucoid adenocarcinoma & $5(16.6 \%)$ \\
of neoplasm & Signet ring carcinoma & $5(16.6 \%)$ \\
& Moderately differentiated adenocarcinoma & $17(56.6 \%)$ \\
& Poorly differentiated adenocarcinoma & $3(10 \%)$ \\
\hline
\end{tabular}


Table 4. Diagnostic performance of Body Fat Composition determinants for CRC Prediction, analyzed as the area under the curve.

\begin{tabular}{lcccc}
\hline & AUC* $^{*}$ & $\mathbf{9 5 \%} \mathbf{C l}^{+}$ & SE** $^{* *}$ & P-value $^{\mathbf{5}}$ \\
\hline Visceral Fat Volume & 0.391 & $0.266-0.515$ & 0.063 & 0.103 \\
Superficial Fat Volume & 0.539 & $0.411-0.668$ & 0.066 & 0.258 \\
Body Fat Rate & 0.375 & $0.209-0.506$ & 0.076 & 0.071 \\
Visceral Fat Rate & 0.630 & $0.469-0.791$ & 0.082 & 0.099 \\
V/S Fat Rate & 0.726 & $0.583-0.869$ & 0.073 & 0.004 \\
Psoas Muscle Vol. & 0.553 & $0.349-0.712$ & 0.081 & 0.504 \\
Obesity Index & 0.427 & $0.273-0.580$ & 0.078 & 0.353 \\
Leptin & 1.000 & $1.000-1.000$ & 0.000 & $<0.001$ \\
Resistin & 0.000 & $0.000-0.000$ & 0.000 & $<0.001$ \\
\hline
\end{tabular}

*AUC $=$ Area under the Curve

$* * \mathrm{SE}=$ Standard Error $+\mathrm{Cl}=$ Confidence Interval

$\$$ Null hypothesis: true area $=0.5$

Table 5. CRC Predictors among the studied Cohort: Multivariate Logistic Regression

\begin{tabular}{lccc}
\hline Variable & OR & 95\% Cl & P-value \\
\hline Age/years & 1.002 & $0.976-1.028$ & 0.908 \\
Sex (Male) & 1.027 & $0.414-2.546$ & 0.954 \\
BMI & 1.024 & $0.987-1.075$ & 0.336 \\
V/S Fat Rate & 0.934 & $0.807-1.080$ & 0.335 \\
Psoas Muscle Vol./cm 3 & 0.998 & $0.994-1.004$ & 0.305 \\
Obesity Index (Too Fat) & 1.704 & $0.665-4.366$ & 0.267 \\
Leptin (ng/ml) & 0.556 & $0.408-0.757$ & $<0.001$ \\
Resistin (ng/ml) & 3.129 & $1.070-7.824$ & 0.009 \\
\hline
\end{tabular}

OR=Odds Ratio; $\mathrm{Cl}=$ Confidence Interval

Although some studies reported reduced levels of serum Leptin in CRC patients (Kumor et al., 2009; Sălăgeanu et al., 2010), others indicated that increased serum Leptin was associated with the incidence of CRC (Stattin et al., 2004), likely attributed to the expression of Leptin receptors in normal human colon mucosa, adenomas, and carcinomas (Hardwick et al., 2001).

In agreement with current study results, Kumor et al. 2009 found lower serum Leptin levels in patients with adenomas compared to control subjects. They suggested that low serum Leptin level in adenoma patients could be linked with some causal factors of hyperLeptinemia and some mechanisms which could not be explained clearly (Kumor et al., 2009).

In the present study, it was observed that circulating Resistin levels were significantly increased in CRC patients as compared with the adenoma and control groups. The current results were in accordance with other studies that found higher levels of Resistin in patients with CRC (Gonullu et al., 2010). Sălăgeanu et al. 2010 reported that although serum Resistin levels increased in CRC patients and their study had no significant correlation between its level and the tumor stage, localization, or grade of differentiation. The current work agreeing with the results of Demir et al., 2015 results could not find a substantial difference in serum Resistin levels between the adenoma and control groups.

Geographical regions, ethnicity, and genetic features may lead to variations in the clinical manifestations of obesity and its complications. Likewise, adipocytokines can show changes(Rea and Donnelly, 2004; Koerner et al., 2005; Otake et al., 2005; Wasim et al., 2006). Therefore, data from work in the Middle East should also be taken into consideration. In agreement with the current results, a study conducted on Turkish patients (Gonullu et al., 2010) investigated the associations between Leptin, Resistin, insulin resistance, and CRC. They found that Resistin levels were significantly higher, and Leptin levels were significantly lower in patients with colorectal cancer compared with controls. CT is having high accuracy and reproducible results among the different assessment tools of adiposity evaluation (Silva et al., 2020). 
The results of current study confirmed the results of Hassan et al. 2013, when stating that BMI was not a predictor of CRC. Moreover, imaging assessment of visceral adiposity also proved to be more accurate than BMI in predicting other crucial factors for patient outcomes such as operative time and surgical complications in gastrointestinal cancers, including CRC. (Silva et al., 2020).

Several studies analyzed the influence of the abdominal fat area in CRC outcomes. Abdominal CT scan images retrieved at the levels of the lumbar vertebrae L3-L4 and L4-L5 were subjected to image analysis to obtain Visceral fat volume, subcutaneous fat volume, and V/S fat ratio and patients were compared in terms of other prognostic but not diagnostic factors including survival, clinical and pathological features. Patients with a high $\mathrm{V} / \mathrm{S}$ fat ratio had a significantly correlation with tumor size, with no differences in Overall survival or other pathological features.(Moon et al., 2008; Silva et al., 2020).

On the other hand, Guiu et al. 2010 reported that high visceral fat areas could predict the poor prognosis and the possibility of occurrence of malignancy. In the current study, the most critical parameter was the $\mathrm{V} / \mathrm{S}$ fat rate with the highest accuracy in differentiating CRC from control persons,. this study was performed on the whole abdominal fields to reach a more precise evaluation, quantification and stratification of adiposity.

Also, our findings were consistent with Bardou et al. 2013, who reported that overweight and obesity were among the leading cause of overall mortality. The association of CRC was evident with obesity and increased fat rate in the body. On the other hand, the current results oppose their results when stating that visceral fat to be of more significant concern than subcutaneous fat. In contrast, in our study, the body fat rate did not have a considerable burden with low accuracy, while the visceral fat was significant between the three groups of the current study with limited power in differentiating CRC from adenoma.

Conversely, these results were in disagreement with Choe et al. 2016, who concluded that there was a significant association between colorectal cancer and subcutaneous fat deposition and also considered visceral fat as a predictor of CRC prognosis. The current study combined these values in $\mathrm{V} / \mathrm{S}$ rate as most eminent parameter in the prediction of CRC. This disagreement is likely attributed to the different methods of evaluation as we incorporated quantitative parameters assessment based on whole abdominal field scan rather than a few scans opposite L3-L5 vertebrae as utilized in the studies as mentioned earlier.

These Controversial findings supported the statement of Hopkins et al. 2018 that the current literature studies are heterogeneous with variable statistical methodology and different cut-off points in defining body composition parameters. Still, the available evidence is not entirely concordant as different studies resulted in distinctive findings in terms of imaging findings and their correlation with survival (Silva et al., 2020). Possible reasons for the inconsistencies found could be related to the use of different methods to measure the Visceral fat volume, subcutaneous fat volume, and $\mathrm{V} / \mathrm{S}$ fat ratio, or even the use of different thresholds to stratify adiposity based on CT imaging analysis (Silva et al., 2020).

Skeletal muscle likely operates through physiologic and metabolic (e.g., inflammation) as well as behavioral pathways. Skeletal muscle has local autocrine, paracrine, and endocrine effects but also secretes cytokines and other myokines, leading to systemic effects. Therefore, higher levels of muscle may decrease the impact of obesity-induced inflammation, whereas lower levels of muscle could lead to local and systemic inflammation. Previous studies suggest that systemic inflammation may lead to ongoing muscle loss in cancer patients and partially drive the associations with cancer survival (Feliciano and Chen, 2018).

Furthermore, Malietzis et al. 2015 found that reduced muscle mass and visceral obesity (as categorical parameters that are quantified by $\mathrm{CT}$ ) could enable early identification of the CRC high-risk patients to allow early planning of oncology treatment. However, accumulating evidence suggests that low skeletal muscle mass at colorectal cancer diagnosis often referred to as sarcopenia. sarcopenia is highly prevalent 
and associated with cancer recurrence, survival, surgical complications, and treatment-related toxicities (Feliciano and Chen, 2018). Studies using CT quantitative muscle volume estimation included heterogeneous patient populations and variable cut-points to define 'low' skeletal muscle mass, leading to a wide range of prevalence. A meta-analysis combined the results of three small studies, two of which were in metastatic colorectal cancer patients, and reported that low skeletal muscle at colorectal cancer diagnosis was associated with a more than 2-fold risk of overall mortality (hazard ratio $(H R)=2.25 ; 95 \% \mathrm{Cl} 1.63,3.09$ ). (Shachar et al. 2016; Feliciano and Chen 2018; Shirdel et al. 2020).

Previously reported data from the colorectal cancer literature strongly suggest that assessments of body composition and skeletal muscle from CT scans could significantly impact preoperative risk assessment and improve decision-making around selection of candidates for surgery and post-operative treatment with a particular emphasis on its association with cancer recurrence and survival, postoperative complications and treatment-related toxicities. (Shachar et al. 2016; Feliciano and Chen 2018; Shirdel et al. 2020). Shirdel et al. 2020 found that sarcopenia but not adiposity measures were associated with decreased cancer-specific survival in non-metastatic, also early-stage CRC. Sarcopenia measured by CT may thus have potential clinical utility for prognostication, therapeutic decision making, and identification of patients in need of early nutritional or pharmacological anti-sarcopenic intervention. (Shirdel et al. 2020). This opposes the results of the current study as psoas muscle volume was not a valid predictor for occurrence of CRC.

We approve Looijaard et al. recommendations concerning change in body composition with muscle strength, physical performance, and other domains to predict adverse outcomes in patients with CRC, particularly in geriatric patients. This may lead to potential insight into mechanisms of colorectal cancer (Looijaard et al., 2020).

\section{Strengths and Limitations}

Among points of strength was the quantitative evaluation of body fat. The evaluation extended from diaphragmatic copula down to symphysis pubis, while many other studies, as conducted by Hopkin et al. 2018, handled slices at the level of the third lumbar vertebra or level of the umbilicus. Among the limitations encountered in this study, is the lack of correlation with tumor size (T-staging), and tumor grading in patients who had colon cancer.

\section{CONCLUSION}

The current study concluded that Leptin and Resistin were independent predictors of occurrence for CRC. Moreover, the V/S fat rate was most eminent parameter of quantitative fat analysis for the prediction of CRC.

\section{List of abbreviations}

BMI Body mass index

CEA Carcinoembryonic antigen

CRC Colorectal cancer

V/S Visceral/subcutaneous fat rate

\section{Data Availability}

The data used to support the findings of this study are available from the corresponding author upon request.

\section{Conflict of interest}

Authors declare that they have no conflicts of interest. No external funding was employed in the current study.

\section{References}

Atta, H., Abdel-Gawad, E.A., ElAzab, A., Saleh, M., Abbas, W.A., Soliman, R., and Imam, H. (2016). Whole ureteric course delineation assessment using non contrast curved sagittal oblique reformatted CT. Egypt. J. Radiol. Nucl. Med. 47, 1103-1110.

Bardou, M., Barkun, A.N., and Martel, M. (2013). Obesity and colorectal cancer. Gut 62, 933947.

Calle, E.E., and Thun, M.J. (2004). Obesity and cancer. Oncogene 23, 6365-6378.

Calle, E.E., Rodriguez, C., Walker-Thurmond, K., and Thun, M.J. (2003). Overweight, obesity, and mortality from cancer in a prospectively studied cohort of US adults. N. Engl. J. Med. 348, 1625-1638.

Choe, E.K., Park, K.J., Ryoo, S.-B., Moon, S.H., Oh, H.K., and Han, E.C. (2016). Prognostic Impact of Changes in Adipose Tissue Areas after Colectomy in Colorectal Cancer Patients. J Korean Med Sci 31, 1571-1578. 
Demir, N., Ahishali, E., Dolapcioglu, C., Ercan, S., Orçun Kaptanagasi, A., Dabak, R., Yucel, N., Sargin, M., and Uygur Bayramicli, O. (2015). The relationship between serum adiponectin and resistin levels, insulin resistance and colorectal adenomas. Turk J Gastroenterol 26, 20-24.

Drew, J.E. (2012). Molecular mechanisms linking adipokines to obesity-related colon cancer: focus on leptin. Proc. Nutr. Soc. 71, 175-180.

Erarslan, E., Turkay, C., Koktener, A., Koca, C., Uz, B., and Bavbek, N. (2009). Association of visceral fat accumulation and adiponectin levels with colorectal neoplasia. Dig. Dis. Sci. 54, 862868.

Faul, F., Erdfelder, E., Lang, A.-G., and Buchner, A. (2007). G* Power 3: A flexible statistical power analysis program for the social, behavioral, and biomedical sciences. Behav. Res. Methods 39, 175-191.

Feliciano, E.C., and Chen, W.Y. (2018). Clinical implications of low skeletal muscle mass in early-stage breast and colorectal cancer. Proc. Nutr. Soc. 77, 382-387.

Goktas, S., Yilmaz, M.I., Caglar, K., Sonmez, A., Kilic, S., and Bedir, S. (2005). Prostate cancer and adiponectin. Urology 65, 1168-1172.

Gonullu, G., Kahraman, H., Bedir, A., Bektas, A., and Yücel, I. (2010). Association between adiponectin, resistin, insulin resistance, and colorectal tumors. Int. J. Colorectal Dis. 25, 205-212.

Guiu, B., Petit, J.M., Bonnetain, F., Ladoire, S., Guiu, S., Cercueil, J.-P., Krausé, D., Hillon, P., Borg, C., and Chauffert, B. (2010). Visceral fat area is an independent predictive biomarker of outcome after first-line bevacizumab-based treatment in metastatic colorectal cancer. Gut 59, 341-347.

Hardwick, J.C.H., Van Den Brink, G.R., Offerhaus, G.J., Van Deventer, S.J.H., and Peppelenbosch, M.P. (2001). Leptin is a growth factor for colonic epithelial cells. Gastroenterology 121, 79-90.

Hassan, C., Pooler, B.D., Kim, D.H., Rinaldi, A., Repici, A., and Pickhardt, P.J. (2013). Computed tomographic colonography for colorectal cancer screening: risk factors for the detection of advanced neoplasia. Cancer 119, 2549-2554.

Hopkins, J.J., Skubleny, D., Bigam, D.L., Baracos, V.E., Eurich, D.T., and Sawyer, M.B. (2018). Barriers to the Interpretation of Body Composition in Colorectal Cancer: A Review of the Methodological Inconsistency and Complexity of the CT-Defined Body Habitus. Ann. Surg. Oncol. 25, 1381-1394.
Hsing, A.W., Chua Jr, S., Gao, Y.-T., Gentzschein, E., Chang, L., Deng, J., and Stanczyk, F.Z. (2001). Prostate cancer risk and serum levels of insulin and leptin: a population-based study. J. Natl. Cancer Inst. 93, 783-789.

Khandekar, M.J., Cohen, P., and Spiegelman, B.M. (2011). Molecular mechanisms of cancer development in obesity. Nat. Rev. Cancer 11, 886-895.

Koerner, A., Kratzsch, J., and Kiess, W. (2005). Adipocytokines: leptin--the classical, resistin-the controversical, adiponectin--the promising, and more to come. Best Pract. Res. Clin. Endocrinol. Metab. 19, 525-546.

Kumor, A., Daniel, P., Pietruczuk, M., and MałeckaPanas, E. (2009). Serum leptin, adiponectin, and resistin concentration in colorectal adenoma and carcinoma (CC) patients. Int. J. Colorectal Dis. 24, 275-281.

Looijaard, S.M.L.M., Meskers, C.G.M., Slee-Valentijn, M.S., Bouman, D.E., Wymenga, A.N.M., Klaase, J.M., and Maier, A.B. (2020). Computed Tomography-Based Body Composition Is Not Consistently Associated with Outcome in Older Patients with Colorectal Cancer. Oncologist 25, e492.

Malietzis, G., Aziz, O., Bagnall, N.M., Johns, N., Fearon, K.C., and Jenkins, J.T. (2015). The role of body composition evaluation by computerized tomography in determining colorectal cancer treatment outcomes: a systematic review. Eur. J. Surg. Oncol. 41, 186-196.

Miyoshi, Y., Funahashi, T., Kihara, S., Taguchi, T., Tamaki, Y., Matsuzawa, Y., and Noguchi, S. (2003). Association of serum adiponectin levels with breast cancer risk. Clin. Cancer Res. 9, 5699-5704.

Moon, H.-G., Ju, Y.-T., Jeong, C.-Y., Jung, E.-J., Lee, Y.J., Hong, S.-C., Ha, W.-S., Park, S.-T., and Choi, S.-K. (2008). Visceral obesity may affect oncologic outcome in patients with colorectal cancer. Ann. Surg. Oncol. 15, 1918-1922.

Nishii, T., Kono, S., Abe, H., Eguchi, H., Shimazaki, K., Hatano, B., and Hamada, H. (2001). Glucose intolerance, plasma insulin levels, and colon adenomas in Japanese men. Japanese J. Cancer Res. 92, 836-840.

Ohshima, S., Yamamoto, S., Yamaji, T., Suzuki, M., Mutoh, M., Iwasaki, M., Sasazuki, S., Kotera, K., Tsugane, S., and Muramatsu, Y. (2008). Development of an automated 3D segmentation program for volume quantification of body fat distribution using CT. Japanese J. Radiol. Technol. 64, 11771181. 
Otake, S., Takeda, H., Suzuki, Y., Fukui, T., Watanabe, S., Ishihama, K., Saito, T., Togashi, H., Nakamura, T., Matsuzawa, Y., et al. (2005). Association of Visceral Fat Accumulation and Plasma Adiponectin with Colorectal Adenoma: Evidence for Participation of Insulin Resistance. Clin. Cancer Res. 11, 3642 LP -3646.

Paik, S.S., Jang, S.-M., Jang, K.-S., Lee, K.H., Choi, D., and Jang, S.J. (2009). Leptin expression correlates with favorable clinicopathologic phenotype and better prognosis in colorectal adenocarcinoma. Ann. Surg. Oncol. 16, 297303.

Paz-Filho, G., Lim, E.L., Wong, M.-L., and Licinio, J. (2011). Associations between adipokines and obesity-related cancer. Front Biosci 16, 16341650.

Petridou, E., Papadiamantis, Y., Markopoulos, C., Spanos, E., Dessypris, N., and Trichopoulos, D. (2000). Leptin and insulin growth factor I in relation to breast cancer (Greece). Cancer Causes Control 11, 383-388.

Petridou, E., Mantzoros, C., Dessypris, N., Koukoulomatis, P., Addy, C., Voulgaris, Z., Chrousos, G., and Trichopoulos, D. (2003). Plasma adiponectin concentrations in relation to endometrial cancer: a case-control study in Greece. J. Clin. Endocrinol. Metab. 88, 993997.

Rea, R., and Donnelly, R. (2004). Resistin: an adipocyte-derived hormone. Has it a role in diabetes and obesity? Diabetes. Obes. Metab. 6, 163-170.

Renehan, A.G., Soerjomataram, I., Tyson, M., Egger, M., Zwahlen, M., Coebergh, J.W., and Buchan, I. (2010). Incident cancer burden attributable to excess body mass index in 30 European countries. Int. J. Cancer 126, 692-702.

Sălăgeanu, A., Tucureanu, C., Lerescu, L., Caraş, I., Pitica, R., Gangurà, G., Costea, R., and Neagu, S. (2010). Serum levels of adipokines resistin and leptin in patients with colon cancer. J. Med. Life 3, 416-420.

Shachar, S.S., Williams, G.R., Muss, H.B., and Nishijima, T.F. (2016). Prognostic value of sarcopenia in adults with solid tumours: a meta-analysis and systematic review. Eur. J. Cancer 57, 58-67.
Shirdel, M., Andersson, F., Myte, R., Axelsson, J., Rutegård, M., Blomqvist, L., Riklund, K., van Guelpen, B., Palmqvist, R., and Gylling, B. (2020a). Body composition measured by computed tomography is associated with colorectal cancer survival, also in early-stage disease. Acta Oncol. (Madr). 1-10.

Shirdel, M., Andersson, F., Myte, R., Axelsson, J., Rutegård, M., Blomqvist, L., Riklund, K., van Guelpen, B., Palmqvist, R., and Gylling, B. (2020b). Body composition measured by computed tomography is associated with colorectal cancer survival, also in early-stage disease. Acta Oncol. (Madr). 0, 1-10.

Silva, A., Faria, G., Araújo, A., and Monteiro, M.P. (2020). Impact of adiposity on staging and prognosis of colorectal cancer. Crit. Rev. Oncol. Hematol. 145, 102857.

Stattin, P., Lukanova, A., Biessy, C., Söderberg, S., Palmqvist, R., Kaaks, R., Olsson, T., and Jellum, E. (2004). Obesity and colon cancer: does leptin provide a link? Int. J. Cancer 109, 149152.

Summers, R.M., Liu, J., Sussman, D.L., Dwyer, A.J., Rehani, B., Pickhardt, P.J., Choi, J.R., and Yao, J. (2012). Association between visceral adiposity and colorectal polyps on CT colonography. Am. J. Roentgenol. 199, 48-57.

Tudek, B., and Speina, E. (2012). Oxidatively damaged DNA and its repair in colon carcinogenesis. Mutat. Res. Mol. Mech. Mutagen. 736, 82-92.

Tulubas, F., Mete, R., Oznur, M., and Topcu, B. (2013). The role of adipocytokines in colon cancer and adenomas/uloga adipocitokina $u$ kanceru i adenomima debelog creva. J. Med. Biochem. 33, 135-142.

Wasim, H., Al-Daghri, N.M., Chetty, R., McTernan, P.G., Barnett, A.H., and Kumar, S. (2006). Relationship of serum adiponectin and resistin to glucose intolerance and fat topography in south-Asians. Cardiovasc. Diabetol. 5, 10.

Wei, E.K., Giovannucci, E., Fuchs, C.S., Willett, W.C., and Mantzoros, C.S. (2005). Low plasma adiponectin levels and risk of colorectal cancer in men: a prospective study. J. Natl. Cancer Inst. 97, 1688-1694. 